\title{
Atividade física e saúde óssea: princípios fundamentais da resposta a estímulos mecânicos
}

\author{
Caroline Ruschel \\ Alessandro Haupenthal \\ Helio Roesler
Centro de Ciências da Saúde e do Esporte da Universidade do Estado de Santa Catarina (CEFID/UDESC), Florianópolis, SC, Brasil

\begin{abstract}
Resumo: O papel-chave da atividade física no desenvolvimento e na manutenção da massa e da resistência ósseas é amplamente aceito. Este artigo revisa informações acerca das diferentes respostas do tecido ósseo aos estímulos mecânicos, através de consulta ao Portal de Periódicos CAPES, utilizando os unitermos "bone", "exercise", "mechanical loading", "bone mass" e "osteogenic response" e incluindo artigos completos publicados entre 1980 e 2008. Os estudos analisados demonstram que: (1) o osso responde melhor a estímulos dinâmicos comparados a estímulos estáticos; (2) a resposta óssea é maior quando a taxa de aplicação da carga é alta; e (3) a resposta óssea satura rapidamente, sendo o intervalo entre as aplicações de carga é uma estratégia benéfica. Conhecer e aplicar informações como essas, além de assegurar que as rotinas de exercício proporcionem um estímulo suficiente para manter a densidade mineral óssea em um nível apropriado, são importantes para a prevenção de mecanismos de lesão osteomuscular.
\end{abstract}

Palavras-chave: Atividade física. Saúde óssea. Resposta osteogênica.

\section{Physical activity and bone health: fundamental principles of the answer to mechanical loading}

\begin{abstract}
The key role of physical activity in the development and maintenance of bone mass and resistance is widely accepted. This article reviews information about the different answers of bone tissue to mechanical loading, obtained through the "Portal de Periódicos CAPES" database, using the descriptors "bone", "exercise", "mechanical loading", "bone mass" and "osteogenic response", and including full papers published in peer-reviewed journals from 1980 to 2008. The analyzed studies showed that: (1) the bone answers better to dynamic stimuli compared to static stimuli; (2) the bone response is better at higher loading rates; and (3) the bone response saturates quickly, and the insertion of rest intervals between the load applications could be a beneficial strategy. Knowing and using this information, beyond assuring that the exercise routines will provide a sufficient stimulus to maintain subject's bone mineral density in a healthy level, could be very helpful to prevent mechanisms of musculoskeletal injuries.
\end{abstract}

Key Words: Physical activity. Bone health. Osteogenic response.

\section{Introdução}

O osso é um dos tecidos mais metabólicos e dinamicamente ativos no corpo e se mantém em atividade durante toda a vida. Por ser um tecido altamente vascularizado, o osso tem excelente capacidade de se regenerar e de alterar suas propriedades e configurações em resposta às mudanças na demanda mecânica (FROST, 1971; NORDIN; FRANKEL, 2003). De acordo com Burr et al. (2002), o osso é capaz de "sentir" as condições mecânicas a que é submetido e de se adaptar a elas.

Para o crescimento e fortalecimento da massa óssea é necessário que um estresse mecânico mínimo seja aplicado, 0 que garante 0 desenvolvimento e a manutenção da integridade esquelética (HAMILL; KNUTZEN, 1999). Segundo Kannus et al. (1996), muitos fatores intrínsecos (genéticos e hormonais) e extrínsecos (biomecânicos e nutricionais) determinantes da massa óssea têm sido documentados na literatura. Dentre as ações capazes de influenciar esses fatores, o papel-chave da atividade física no desenvolvimento e na manutenção da massa e da resistência ósseas é amplamente aceito (KANNUS et al., 1996; UMEMURA et al., 1997; HUMPHRIES et al., 2000; BURR et al. 2002; SRINIVASAN et al., 2002; WARDEN; TURNER, 2004; MOSER et al., 2004; LORENTZON et al., 2005; CADORE et al., 2005; GROSS; SRINIVASAN, 2006). 
Em um caso de osteoporose, por exemplo, a etiologia da doença inclui fatores como envelhecimento, hormônios, nutrição, genética e carga mecânica através do exercício; desses fatores, apenas a carga dinâmica aumentada estimula comprovadamente a formação óssea (VOLOSHIN, 2004). É importante observar que, em casos como esse, o fator descrito como mais importante para a promoção da formação óssea e para a minimização dos efeitos debilitantes da doença - a aplicação de carga através do exercício - pode ser controlado e manipulado pelos profissionais envolvidos com a profilaxia e reabilitação: os educadores físicos e os fisioterapeutas. Para tanto, os protocolos de exercícios necessitam ser baseados no conhecimento advindo de estudos experimentais e clínicos, a fim de que seja estabelecida uma estratégia segura e eficaz composta por atividades que promovam a adaptação desejada na massa e na resistência dos ossos, sem colocar em risco a integridade musculoesquelética.

O sucesso de todo e qualquer programa de exercícios físicos depende da aplicação de princípios que consideram fatores como a individualidade biológica, as características da sobrecarga aplicada, a interdependência volumeintensidade e 0 período de recuperação necessário para a adaptação do organismo (DANTAS, 1995; BOMPA, 1998; WEINECK, 1999). Em se tratando do desenvolvimento de protocolos de exercícios que tenham por objetivo a manutenção e/ou o aumento da massa óssea, isso não é diferente.

Entretanto, apesar do consenso a respeito da relação positiva entre a atividade física e os processos de manutenção e ganho de massa óssea, muitas questões interessantes relativas à adaptação do osso aos exercícios ainda não foram respondidas, porque são insuficientes as informações sobre o tipo, a intensidade, a frequência e a duração ideais para a promoção do máximo estímulo anabólico ao osso. Kannus et al. (1996), em trabalho escrito em 1996, afirmam que a partir do conhecimento adquirido até então, um protocolo ideal deveria (a) estimular mecanicamente o osso-alvo com altos picos de força e altas taxas de aplicação de carga (alto impacto), (b) criar distribuições versáteis de tensão através da estrutura óssea (como por exemplo, estimular o osso em direções às quais ele não está acostumado), (c) consistir de repetições e sessões de treinamento relativamente curtas e (d) ser de natureza longitudinal e progressiva.

As orientações propostas acima vêm sendo discutidas ao longo da última década. Com certeza, a primeira delas, que propõe que o osso seja estimulado com cargas de grande magnitude e com uma alta freqüência, recebeu maior atenção por parte dos pesquisadores da área. Isso porque, apesar de servir para aumentar a força do esqueleto e constituir um meio potencial de profilaxia de futuras doenças, cargas de alto impacto não são praticáveis para aquelas pessoas que mais são acometidas por osteopenias e por isso necessitam de um aumento de massa óssea (VOLOSHIN, 2004). Exercícios que são acessíveis para esse tipo de população, como aqueles em que a carga relativa do peso corporal atua sobre o esqueleto de forma moderada (caminhada e exercícios de resistência, por exemplo), não são percebidos como estímulos suficientes para desencadear um processo de formação óssea.

Por isso, a tentativa de estabelecer protocolos de intervenção específicos continua a ser uma área de substancial estudo (GROSS; SRINIVASAN, 2006), e os esforços recentes têm sido focados no desenvolvimento de estratégias para "enganar" o osso e fazer com que ele "perceba" os exercícios de carga moderada como estímulos suficientes para desencadear a produção de tecido ósseo (VOLOSHIN, 2004). Essas estratégias partem do princípio de que uma determinada carga possui muitos atributos: ela não é definida apenas por sua magnitude, mas também por suas características dinâmicas, como a frequência e o volume (duração) de sua aplicação (McGINNIS, 2002). Sendo assim, vários estudos têm investigado as conseqüências da modificação dessas características e os dados sugerem que elas interagem umas com as outras (RUBIN; LANYON, 1984; TURNER, 1998; BURR et al., 2002; OCARINO; SERAKIDES, 2006).

Com base nas informações supracitadas, esta revisão teve por objetivo reunir informações acerca das diferentes respostas do tecido ósseo aos estímulos mecânicos, a fim de fornecer subsídios para a estruturação de programas de exercícios ideais para a otimização do processo de formação/manutenção da massa óssea. Para tanto, foi realizada uma consulta a artigos completos publicados em periódicos nacionais e 
internacionais, acessados por meio da base de dados Portal Periódicos CAPES, utilizando os unitermos "bone", "exercise", "mechanical loading", "bone mass" e "osteogenic response". Foram incluídos nesta revisão artigos na íntegra publicados em português e inglês, no período de 1980 a 2008, que apresentassem informações acerca das seguintes questões norteadoras: "Qual o melhor tipo de aplicação de carga?", "Qual a taxa ideal de aplicação de carga?" e "Qual a importância do volume e da inserção de intervalos na aplicação da carga?". Foram também consultados livros clássicos utilizados em biomecânica, publicados em português e inglês, para a obtenção de informações relevantes ao tema de estudo.

\section{Tipo de carga: os ossos só respondem a estímulos dinâmicos}

O fenômeno da remodelação óssea promovida em resposta às demandas mecânicas, no qual o osso perde ou ganha tecido ósseo esponjoso ou cortical em resposta ao nível de estresse sustentado, é sumarizado como Lei de Wolff, a qual enuncia que a remodelação óssea é influenciada e modulada pelo estresse mecânico (NORDIN; FRANKEL, 2003).

De acordo com Burr et al. (2002), a idéia que o osso sente as condições mecânicas a que é submetido e que pode se adaptar a elas não é novidade; entretanto, os sinais mecânicos específicos detectados pelas células e a maneira pela qual estes sinais são convertidos em atividade celular que resulta na remodelação óssea, ainda constituem hipóteses teóricas a serem confirmadas.

A força mecânica, quando aplicada sobre o tecido ósseo, gera sinais endógenos que interferem nos processos de remodelação óssea; esses sinais são captados por um sistema mecanosensorial no qual o osteócito é a principal célula responsável por traduzir a força mecânica em sinais bioquímicos que regulam a modelação (OCARINO; SERAKIDES, 2006). Segundo Frost (1971), as tensões aplicadas na superfície óssea são convertidas em sinais que guiam tipos especiais de célula - os osteócitos - que, quando ativadas, ajustam o tamanho e a geometria do osso de uma maneira que minimizará futuras tensões.

Thompson (apud BURR et al., 2002), apontou a deformação como um sinal mecânico que controla a adaptação dos ossos, e propôs que cargas de cisalhamento, produzidas como resultado dessa deformação, podem ser componentes do mecanismo de sinalização mecânica. Sugere-se que a deformação produz estes efeitos indiretamente ao causar um movimento de fluido para dentro dos espaços ao redor dos osteócitos e dos processos canaliculares, criando uma carga de cisalhamento através do fluxo laminar do fluido nos osteócitos, ao invés de provocar uma deformação direta da membrana celular.

É importante considerar que o fluido pode ser movido dentro do osso apenas com a aplicação de cargas cíclicas seguidas de relaxamento; cargas estáticas não irão causar esse movimento e nem irão promover as cargas de cisalhamento necessárias na célula para gerar uma resposta anabólica adaptativa. Se a adaptação é determinada pelas cargas de cisalhamento nas células ósseas, então esta só deve ocorrer em resposta a cargas dinâmicas (BURR et al., 2002).

Desde os estudos de Hert e Liskova, na década de 70, sabe-se que o osso se adapta apenas em resposta a cargas dinâmicas e não a cargas estáticas (BURR et al., 2002). Mais recentemente, há evidências de que cargas estáticas não só não produzem resposta, como podem suprimir o crescimento normal. Robling et al. (2001) submeteram um grupo de ratos a sessões de cargas de $10 \mathrm{~min} /$ dia durante 2 semanas, utilizando 3 condições diferentes de aplicação (estática, com magnitude de $8,5 \mathrm{~N}$, estática com magnitude de $17 \mathrm{~N}$ e dinâmica com magnitude de $17 \mathrm{~N}$ ). Os autores observaram que a aplicação dinâmica da carga aumentou a significativamente a osteogênese nas superfícies periosteal e endocortical, enquanto que a carga estática não teve efeito sobre a taxa de formação óssea endocortical e suprimiu a formação óssea periosteal. Desta forma, as evidências encontradas nesse estudo mostram que cargas dinâmicas são necessárias não só para estimular - crescimento ósseo, mas aparentemente também para prevenir a supressão desse crescimento.

É importante ressaltar que embora a carga dinâmica no sistema musculoesquelético humano seja claramente benéfica devido à sua contribuição para o aumento da densidade mineral óssea, ao mesmo tempo ela pode levar ao desenvolvimento de distúrbios 
musculoesqueléticos. Dessa forma, é de grande importância desenvolver metodologias cientificamente razoáveis para avaliar o limite superior (que definirá o limite de excitação dinâmica que pode ser suportado pelo organismo sem o desenvolvimento de lesões) e o limite inferior (que definirá a excitação dinâmica mínima exigida pelo osso para manter um nível saudável de conteúdo mineral) seguros e úteis de aplicação da carga dinâmica (VOLOSHIN, 2004).

\section{A taxa de aplicação de carga é crítica para a resposta óssea}

De acordo com Burr et al. (2002), o fato de que cargas cíclicas e dinâmicas são necessárias para iniciar uma resposta adaptativa sugere que a resposta óssea depende de outros fatores além da magnitude da tensão aplicada. Nordin e Frankel (2003) afirmam que, uma vez que o osso é um material viscoelástico, seu comportamento biomecânico varia com a razão na qual é carregado (modo de aplicação da carga). Segundo McGinnis (2002), a taxa de aplicação da carga, determinada pela magnitude e pela freqüência, é um fator importante a ser considerado, pois afeta a força mecânica e a rigidez do osso.

Turner et al. (1994) e Turner et al. (1995) demonstraram em seus estudos com tíbias de ratos que a taxa de aplicação da carga mantém uma relação de causa-efeito com o processo de adaptação óssea. Anos mais tarde, Turner (1998) deduziu que essa taxa é diretamente proporcional à magnitude e a freqüência de aplicação da carga. Dessa forma, uma importante consideração vem à tona: para aumentar o estímulo de tensão e como conseqüência a resposta óssea, aumenta-se a freqüência de aplicação da carga e, dessa forma, a magnitude pode ser mantida constante. Ainda neste mesmo ano, Mosley e Lanyon (1998) mostraram que mudanças na taxa de aplicação de carga promovem um estímulo osteogênico maior do que mudanças na magnitude da carga.

Vale ressaltar que nos estudos de Turner foi observado um aumento significativo da taxa de formação óssea em freqüências variando de $0,5 \mathrm{a}$ $2,0 \mathrm{~Hz}$, numa magnitude de carga constante (TURNER et al., 1994; TURNER et al., 1995). Essa idéia de que a freqüência de aplicação da carga afeta a relação dose-resposta entre as condições mecânicas e a resposta adaptativa é um conceito bastante útil para propósitos práticos (BURR et al., 2002).

O conhecimento da relação proporcional direta entre a frequência e o estímulo osteogênico fez com que outros estudos fossem realizados desde então. Uma informação simples começou a ser questionada: até quando a relação entre freqüência e resposta é linear? Numa tentativa de responder a essa pergunta, Warden e Turner (2004) realizaram um estudo que teve por objetivo investigar a adaptação óssea a cargas aplicadas com freqüências de até $30 \mathrm{~Hz}(1,5,10,20$ e 30 $\mathrm{Hz}$ ), em duas situações diferentes: (a) ratos submetidos a 120 ciclos de carga/dia durante 3 dias e (b) ratos submetidos a 5 minutos de aplicação de carga/dia durante 4 semanas. Tanto para o controle do número de ciclos como da duração de aplicação da carga, os maiores valores de taxa de formação óssea foram obtidos quando utilizada a freqüência de $10 \mathrm{~Hz}$, em comparação às freqüências de 1 e $5 \mathrm{~Hz}$. Além disso, cargas aplicadas a $20 \mathrm{~Hz}$ e $30 \mathrm{~Hz}$ resultaram numa formação óssea reduzida em relação àquela alcançada com a aplicação de cargas a $10 \mathrm{~Hz}$. Estes achados sugerem que a resposta óssea ao aumento da freqüência de aplicação da carga não é infinitamente linear, e que nenhum benefício adicional pode ser promovido com a aplicação de cargas a freqüências maiores do que $10 \mathrm{~Hz}$.

Essas informações observadas nos últimos anos contrastam com a crença inicial de que magnitudes de tensão crescentes consistiam no método ótimo de estimular a osteogênese (VOLOSHIN, 2004). A descoberta que tensões baixas são osteogênicas quando aplicadas em altas taxas é a primeira estratégia a ser utilizada para que possamos adequar os protocolos de intervenção às características peculiares da população atendida.

A resposta óssea satura rapidamente: a importância do volume e da inserção de intervalos

Segundo Burr et al. (2002), uma propriedade do processo de resposta óssea tem se tornado cada vez mais evidente: a resposta osteogênica satura rapidamente em resposta às cargas mecânicas. As células necessitam de um período de recuperação para restabelecer a sua sensibilidade mecânica antes de estarem totalmente prontas para responder novamente a estímulos mecânicos. Se a resposta óssea satura 
rapidamente, faz sentido que se pergunte qual o volume de aplicação de carga que leva à saturação e quanto tempo é necessário para que o osso recupere sua sensibilidade mecânica.

Em relação ao volume, os estudos têm demonstrado que o aumento da duração da sessão de carga (número de ciclos ou tempo de aplicação da carga) resulta num retorno diminuído na formação óssea, sugerindo que as células se tornam pouco ou nada sensíveis a estímulos mecânicos repetitivos, desencadeando um mecanismo chamado de saturação mecanosensorial (BURR et al., 2002).

Há cerca de duas décadas, Rubin e Lanyon (1984), ao investigar a resposta óssea na ulna de aves, mostraram que apenas 36 ciclos/dia de

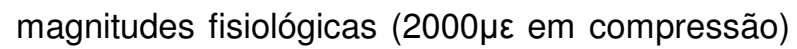
foram tão efetivos na promoção de formação óssea quanto 1800 ciclos/dia com a mesma magnitude. A magnitude da resposta óssea não foi aumentada pelos ciclos adicionais além dos 36 ciclos, indicando que a resposta celular à carga mecânica satura rapidamente. Resultado semelhante foi obtido por Umemura et al. (1997), em seu estudo com ratos, sugerindo que poucos ciclos de carga são necessários para saturar a resposta óssea. Nesse estudo os autores mostraram que apenas 5 saltos/dia foram suficientes para causar um aumento significativo na área cortical e na resistência à flexão e que, a partir de 10 até 100 saltos/dia, não foram encontradas melhoras na taxa de formação óssea.

Em relação ao tempo necessário para que o osso volte a responder aos estímulos de maneira eficaz, vários autores têm observado que a inserção de intervalos de repouso entre os eventos de aplicação de carga aumenta consideravelmente a resposta do osso à carga (ROBLING et al., 2000; SRINIVASAN; GROSS, 2000; BURR et al., 2002; SAXON et al., 2005). De acordo com Gross e Srinivasan (2006), essa estratégia é capaz de transformar um breve protocolo (por exemplo, com duração de 100 segundos) de baixa magnitude, que é normalmente ignorado pelo osso, em um processo osteogênico em potencial.

Para determinar o volume de cada sessão diária de carga e o intervalo ideal entre as sessões, Robling et al. (2000) relataram, em um estudo no qual ratos foram submetidos a diferentes protocolos, que ao permitir um período de recuperação de no mínimo 2-3 horas entre cada sessão de ciclos de carga, promove-se um estímulo osteogênico maior do que quando se aplicam todos os ciclos de carga de uma só vez; e ainda, as células ósseas se tornam significativamente menos sensíveis à carga aplicada após cerca de 60 ciclos.

Os mesmos autores, num outro experimento, buscaram verificar qual o período ideal requerido para a recuperação ótima da sensibilidade mecânica, variando o intervalo de tempo entre sessões idênticas de 90 ciclos/dia, e verificaram que a resposta óssea pode ser melhorada com um período de 4-8 horas de recuperação entre as sessões de aplicação de cargas.

Outra questão foi levantada pelos estudiosos da área: a influência de pequenos intervalos entre os ciclos de uma mesma sessão na taxa de formação óssea. Srinivasan e Gross (2000) submeteram dois grupos de perus a diferentes tipos de sessão de cargas $(f=1 \mathrm{~Hz})$ : (a) 100 ciclos/dia, sem intervalo entre os ciclos; e (b) 100 ciclos/dia com intervalo de 10 segundos entre os ciclos. Os resultados mostraram que a taxa de formação óssea do grupo "b" foi significativamente maior do que do grupo "a". Os achados sugerem que a introdução de um período de intervalo de $10 \mathrm{~s}$ entre os ciclos de carga transformaram um regime de baixa-carga num potente estímulo anabólico.

Neste mesmo sentido, Robling et al. (2000) submeteram ratos a sessões de 36 ciclos de carga $(54 \mathrm{~N}, 2 \mathrm{~Hz})$ com intervalos diferentes entre os ciclos: (a) 0,5 segundos de intervalo entre os ciclos; (b) 3,5 segundos de intervalo entre os ciclos; (c) 7 segundos de intervalo entre os ciclos; (d) 14 segundos de intervalo entre os ciclos; (e) grupo controle com carga simulada, sem intervalo; e (f) grupo controle sem aplicação de carga. Os resultados mostraram que a mineralização superficial e a taxa de formação óssea dos grupos "a", "b" e "c" foi significativamente maior do que dos grupos "e" e "f". O grupo "d", além de apresentar mineralização superficial e taxa de formação óssea significativamente maior do que os grupos "e" e "f", demonstraram um aumento de $50 \%$ destas variáveis em relação aos grupos "a", "b" e "c". Os dados indicam que, além de intervalos entre as sessões diárias de ciclos, um intervalo maior 
entre os ciclos de uma única sessão podem melhorar a resposta osteogênica.

Sob outro ponto de vista, também com o objetivo de investigar a influência dos períodos de recuperação sobre a formação óssea, Saxon et al. (2005) analisaram 56 ratos, divididos em 5 grupos (3 grupos foram submetidos a 360 ciclos/dia de $15 \mathrm{~N}$ e freqüência de $2 \mathrm{~Hz}$ durante 15 semanas; e 2 não foram submetidos - grupos controle). No grupo 1, a carga foi aplicada nas primeiras 5 semanas, seguidas de 10 semanas de repouso; no grupo 2, a carga foi aplicada nas 5 primeiras e nas 5 últimas semanas, com 5 semanas de intervalo entre elas; e no grupo 3, foram aplicadas cargas durante as 15 semanas. Os resultados mostraram que a realização de exercícios durante 5 semanas foi tão efetivo quanto durante 15 semanas na massa e na geometria óssea. Entretanto, reiniciar o exercício após um período de 5 semanas de repouso (grupo 2), promove uma melhora na sensibilidade mecânica e aumenta o ganho na formação óssea e na resistência à fratura, quando comparado com cargas aplicadas continuamente durante 15 semanas.

Dessa forma, parece que a inserção de períodos de repouso serve para reduzir a quantidade e a magnitude da carga necessárias em uma intervenção para que ela seja percebida como estímulo (GROSS; SRINIVASAN, 2006). Vale lembrar também que, desde que ossos vivos são auto-regeneráveis, lesões como a fratura por fadiga ocorrem somente quando o processo de remodelação é ultrapassado pelo processo de fadiga, isto é, quando o volume e a frequência da carga são exagerados e não respeitam o período de recuperação, impedindo a remodelagem necessária para prevenir a falha (NORDIN; FRANKEL, 2003).

\section{Conclusões e Implicações}

Conforme evidenciado, o exercício regular produz efeitos na densidade, no tamanho e na forma dos ossos, resultando em melhoras substanciais na resistência mecânica. Apesar de ainda hoje não haver um claro consenso sobre como exatamente um indivíduo deve ser exercitar no intuito de colher as melhores respostas em termos de saúde óssea, é imprescindível que as informações advindas dos estudos com animais sejam discutidas e analisadas de forma crítica, buscando adaptações que permitam sua utilização quando aplicadas a seres humanos.
Sob uma perspectiva de saúde pública, os profissionais da saúde devem incentivar a prática de atividades físicas desde a infância e puberdade até a idade adulta e senil. Entretanto, ao escolher um determinado exercício, é importante ter em mãos informações capazes de responder perguntas como "quanta carga é aplicada?", “onde a carga é aplicada?", "por quanto tempo a carga é aplicada", "quantas vezes a carga é aplicada?", "a magnitude da carga é constante ou variável?" e "com que rapidez a carga é aplicada?".

Essas informações, além de assegurar que as rotinas de exercício proporcionem um estímulo suficiente para manter a densidade mineral óssea em um nível apropriado, são extremamente importantes para a prevenção de mecanismos de lesão osteomuscular. Protocolos de intervenção com base em informações como aquelas apresentadas no decorrer do texto são ferramentas bastante úteis nas rotinas de trabalho de profissionais da área da saúde, principalmente os educadores físicos e os fisioterapeutas, cujas ações são geralmente baseadas no conhecimento prático adquirido no dia-a-dia. Esses protocolos, no entanto, devem ser específicos para a população à que se destinam, tendo em vista que os processos de adaptação são influenciados pela idade, sexo, natureza debilitante das patologias relacionadas à perda óssea e também pelo contexto biomecânico no qual o esqueleto está inserido (forças externas, forças internas, articulações, tendões, ligamentos e músculos).

\section{Referências}

\section{BOMPA, T. Teoria e metodologia do}

treinamento. Rio de Janeiro: Manole, 1998.

BURR, D. B.; ROBLING, A. G.; TURNER, C. H. Effects of biomechanical stress on bones in animals. Bone, v.30, n.5, 2002. p. 781-786. doi:10.1016/S8756-3282(02)00707-X

CADORE, E. L.; BRENTANO, M. A.; KRUEL, L. F. $M$. Efeitos da atividade física na densidade mineral óssea e na remodelação do tecido ósseo. Revista Brasileira de Medicina do Esporte, v. 11 , n. 6, 2005. p. 373-379. doi:10.1590/S1517$\underline{86922005000600013}$

DANTAS, E. H. M. A prática da preparação

física. $3^{\underline{a}}$ ed. Rio de Janeiro: Shape, 1995. 321p.

FROST, H. M. An introduction to

biomechanics. 2. ed. Springfield: Charles e Thomas, 1971. 151p. 
GROSS, T. S.; SRINIVASAN, S. Building bone mass through exercise: could less be more? British Journal of Sports Medicine, v.40, 2006. p. 2-3. doi: 10.1136/bjsm.2004.016972

HAMILL, J.; KNUTZEN, K. Bases biomecânicas do movimento humano. São Paulo: Manole, 1999. $532 \mathrm{p}$.

HUMPHRIES, B.; NEWTON, R. U.; BRONKS, R.; MARSHALL, S.; McBRIDE, J.; McBRIDE, T. T., et al. Effect of exercise intensity on bone density, strength, and calcium turnover in older women. Medicine and Science in Sports and Exercise. v. 32, 2000. p. 1043-1050. Disponível em: www.ncbi.nlm.nih.gov/pubmed/10862528. Acesso em 12 set 2007.

KANNUS, P.; SIEVÄNEN, H.; VUORI. I. Physical loading, exercise and bone. Bone, v.18, n.1, 1996. p. 1S-3S. doi:10.1016/8756-3282(95)00372$\underline{x}$

LORENTZON, M.; MELLSTRÖM, D.; OHLSSON, C. Association of amount of physical activity with cortical bone size and trabecular volumetric bmd in young adult men: the good study. Journal of Bone and Mineral Research. v. 20, n. 11, 2005. p. 1936-1943. http://dx.doi.org/10.1359/JBMR.050709

McGINNIS, P. M. Biomecânica do esporte e exercício. Porto Alegre: Artmed, 2002. p. 403.

MOSER, D. C.; MELO, S. I. L.; SANTOS, S. G. Influência da atividade física sobre a massa óssea de mulheres. Revista Brasileira de

Cineantropometria e Desempenho Humano. v. 6, n. 1, 2004. p. 46-53. Disponível em: www.rbcdh.ufsc.br/DownloadArtigo.do?artigo $=111$ . Acesso em 06 jul 2008.

MOSLEY, J. R.; LANYON, L. E. Strain rate as a controlling influence on adaptive modeling in response to dynamic loading of the ulna in growing male rats. Bone, v.23, 1998. p. 313-318. doi:10.1016/S8756-3282(98)00113-6

NORDIN, M.; FRANKEL, V. H. Biomecânica básica do sistema musculoesquelético. Rio de Janeiro: Guanabara Koogan, 2003.

OCARINO; N. M.; SERAKIDES, R. Efeito da atividade física no osso normal e na prevenção e tratamento da osteoporose. Revista Brasileira de Medicina do Esporte, v.12, n.3, 2006. p. 164168. Disponível em: www.scielo.br/pdf/rbme/v12n3/v12n3a11.pdf . Acesso em 06 jul 2008.

ROBLING, A. G.; BURR, D. B.; TURNER, C. H. Partitioning a daily mechanical sitimulus intro discrete loading bouts improves the osteogenic response to loading. Journal of Bone and Mineral Research, v.15, 2000. p. 1596-1602. doi:10.1016/S8756-3282(02)00871-2

RUBIN, C. T.; LANYON, L. E. Regulation of bone formation by applied dynamic loads. Journal of Bone and Surgery, v.29, 1984. p. 397-402.

SAXON; L. K.; ROBLING, I. A.; TURNER, A. H. Mechanosensitivity of the rat skeleton decreases after a long period of loading, but is improved with time off. Bone, v.36, 2005. p. 454-464. doi:10.1016/j.bone.2004.12.001

SRINIVASAN; S.; GROSS, T. Intermittent rest enhances osteoblastic activation induced by mechanical loading. Orthopaedic Research Society, v.25, 2000. p. 628-630.

SRINIVASAN; S.; WEIMER, D. A.; AGANS, S. C.; BAIN, S. D.; GROSS, T. Low-magnitude mechanical loading becomes osteogenic when rest is inserted between each load cycle. Journal of Bone and Mineral Research, v.9, n.9, 2002. p. 1613-1620. Disponível em:

http://www.jbmronline.org/doi/full/10.1359/ibmr.20 02.17 .9 .1613 ?cookieSet $=1$ Acesso em 04 out 2008.

TURNER, C. H. Three rules for bone adaptation to mechanical stimulli. Bone, v.23, 1998. p. 399-407. doi:10.1016/S8756-3282(98)00118-5

TURNER, C. H.; FORWOOD, M. R.; OTTER, M. $W$. Mechanotransduction in bone: role of strain rate. American Journal of Physiology, v.269, 1994. p. 875-878.

TURNER, C. H.; OWAN, I.; TAKANO, Y. Mechanotransduction in bone: role of strain rate. American Journal of Physiology, v.269, 1995. p. 438-432.

UMEMURA, Y.; ISHIKO, T.; YAMAUCHI, T.; KURONO, M.; MASHIKO, S. Five jumps per day increase bone mass and breaking force in rats. Journal of Bone and Mineral Research, v.12, 1997. p. 1480-1485. doi: 10.1359/jbmr.1997.12.9.1480

VOLOSHIN, A. S. Propagação do impacto e seus efeitos sobre o corpo humano. In: ZATSIORSKY, V. M. (Ed.). Biomecânica no esporte: performance do desempenho e prevenção de lesão. Rio de Janeiro: Guanabara Koogan, 2004. p. $452-460$.

WARDEN, S. J.; TURNER, C. H.

Mechanotransduction in cortical bone is most efficient at loading frequencies of $5-10 \mathrm{~Hz}$. Bone, 
v.34, 2004. p. 261-270.

doi:10.1016/i.bone.2003.11.011

WEINECK, J. Treinamento ideal. 9. ed. São

Paulo: Manole, 1999. 740p.

Endereço:

Caroline Ruschel

R. Desembargador Pedro Silva, 2100/305-A

Bairro: Coqueiros

Florianópolis SC Brasil

88.080-700

Telefone: (48) 9947-3165

Fax: (48) 3244-2260

e-mail: carolruschel@yahoo.com.br

Recebido em: 08 de abril de 2009.

Aceito em: 21 de dezembro de 2009.

\section{(C)}

Motriz. Revista de Educação Física. UNESP, Rio Claro, SP, Brasil - elSSN: 1980-6574 - está licenciada sob Licenca Creative Commons 